\title{
Termination-dependence of Fermi level pinning at rare-earth arsenide/GaAs interfaces
}

\author{
Zhaofu Zhang ${ }^{1}$, Yuzheng Guo ${ }^{2}$, John Robertson ${ }^{1, *}$ \\ ${ }^{1}$ Department of Engineering, University of Cambridge, Cambridge, CB2 1PZ, UK \\ ${ }^{2}$ College of Engineering, Swansea University, Swansea, SA1 8EN, United Kingdom \\ *Email: jr@eng.cam.ac.uk
}

\begin{abstract}
The properties of metal/semiconductor interfaces are generally described by the metal-induced gap states (MIGS) model. However, rare-earth (RE) arsenide interfaces are found not to follow the MIGS model, in having very different Schottky barrier heights (SBHs) for the Ga- or As-terminations of polar (100) or (111) RE-As/GaAs interfaces. Density function supercell calculations find this effect is due to localized defect interface states located on the mis-coordinated atoms of these interfaces that pin their SBHs at very different energies for each termination, as determined by the anion sublattice bonding. Band offsets of semiconducting $\mathrm{ScN} / \mathrm{GaN}$ interfaces also depend on their termination, as determined by the same defect interface states. This pinning mechanism dominates any MIGS mechanism when it arises. Nonpolar (110) interfaces have little change in bonding, so they have no defect interface states, and we find their SBH is pinned by MIGS at the charge neutrality level. Hence the traditional MIGS models should be extended to include such interface states in a more general description.
\end{abstract}

Pinning of the metal Fermi level $\left(\mathrm{E}_{\mathrm{F}}\right)$ at an energy in the semiconductor band gap at metal/semiconductor interfaces limits the controllability of Schottky barrier heights (SBHs) at these interfaces. This limits our ability to reduce the contact resistance of both traditional and low-dimensional semiconductor devices [1-5]. For many semiconductors with moderate band gap, like $\mathrm{Si}, \mathrm{Ge}, \mathrm{GaAs}$ or transition metal dichalcogenides (TMDs), the SBH varies quite weakly with metal work function, due to a large density of metal-induced gap states (MIGS) at the interface [6-8]. MIGS are the evanescent states of the metal's extended states as they decay into the semiconductor. These states have the same behaviour in most metal/ semiconductor interfaces, and tend to pin $\mathrm{E}_{\mathrm{F}}$ near the semiconductor charge neutrality level (CNL) [6-8]. For $\mathrm{Si}$ or $\mathrm{Ge}, \mathrm{E}_{\mathrm{F}}$ is pinned in the lower gap [6] or near the valence band maximum (VBM) for Ge [9].

There are some exceptions to this strong pinning. The clearest exceptions are metal silicides and germanides [10-14]. For silicides on $\mathrm{Si}$, the SBH varies more strongly with their work function than for elemental metals on Si. This de-pinning effect is accompanied by a strong variation of SBH with $\mathrm{Si}$ face orientation $[11,12]$. The $\mathrm{E}_{\mathrm{F}}$ shift was found to arise from localized 'defect states' near $\mathrm{E}_{\mathrm{F}}$ at mis-coordinated sites along the silicide/Si interface $[15,16]$.

For metals on GaAs and related III-Vs, $E_{F}$ is pinned at an energy which was first identified as a defect level [17,18], and then as the CNL [19,20]. A significant exception to strong pinning in the GaAs system are the metallic rare-earth (RE) arsenides such as ErAs/ GaAs interfaces where the SBH varies with interface orientation from (100), (110) to (111) 
[21]. For a specific orientation, there is an unusually large variation of SBH with the termination, either Ga-first or As-first [21,22]. These exceptions are quite rare so that a special effort should be made to understand its cause, in order to find a better model of SBHs and to understand how to control contact resistances more generally. There have been various studies to find the origin of this behaviour. To date, Lambrecht et al [23] observed that a type of interface state created different pinning energies for each termination. Delaney et al [24] studied the SBH variations in terms of total valence charge densities and an analysis of the local density of states.

Here, we study Fermi-level de-pinning at RE-As interfaces in a different way. We identify the presence of localised interface defect states as in the silicides, and we link the interface state energies directly to their SBH values for each orientation. Experimentally, most studies of REAs/GaAs interfaces have been for rare-earths with f-states [25-27]. Here we use the analogous YAs/GaAs interface system. This simplifies the electronic structure calculations by omitting the computational cost of highly correlated $4 \mathrm{f}$ levels. We can extrapolate to other RE-arsenides by using their bulk band structures. We also extend the analysis to the related semiconducting $\mathrm{ScN} / \mathrm{GaN}$ system.

Our calculations use the CASTEP code [28]. The calculations are converged to a residual energy difference of $10^{-5} \mathrm{eV}$ and force tolerance of $0.02 \mathrm{eV} / \AA$. The primitive cell band structures of YAs were calculated by the screened exchange (SX) hybrid functional [29]. SX can also treat f-shells where necessary [30]. For the interface calculations we used the generalised gradient approximation (GGA) as the electronic exchange-correlation functional and ultra-soft pseudopotentials with a plane-wave cut-off energy of $300 \mathrm{eV}$. The (110) nonpolar interface supercells contain 9 layers of RE arsenide and 9 layers of GaAs, while the (100) supercells consist of 9 layers of RE arsenide and 15 layers of GaAs. A supercell slab with $(\sqrt{2} \times \sqrt{2})$ in-plane periodicity is used, to give an even number of electrons and a spinless system. A $5 \times 5 \times 1 \mathrm{k}$-point mesh giving $13 \mathrm{k}$-points was adopted. The supercells contained two interfaces with no vacuum layer. The supercell lattices were relaxed along $z$ direction to release any internal stress. Other different relaxation schemes of cell vectors were also compared to test the impact of relaxation on SBH. It turns out that the energy difference is within $40 \mathrm{meV} / \mathrm{cell}$, and they have similar PDOS curves and SBHs. So the relaxation detail does not have much effect on the defect-induced EF pinning energies.

Metal arsenides like YAs have two phases, a semiconducting zincblende (zb) phase and a metallic rocksalt (rs) phase (Fig. 1). The rs-YAs is $0.3 \mathrm{eV}$ per formula unit more stable than its zb-phase. The lattice constants of rs-YAs and zb-YAs are $5.83 \AA$ and $5.75 \AA$, respectively. Our calculated lattice constant of GaAs is $5.78 \AA$, a $1 \%$ mismatch to rs-YAs. The growth of GaAs on rocksalt RE-As was studied previously with good lattice-matching [24-26]. Ion channelling shows that the As sublattice is continuous across the interface [25]. STEM lattice images [22] show two interface geometries for the polar (100) orientation, a Ga-terminated interface (Fig. 2(a)) and an As-terminated interface (Fig. 2(b)).

The interface formation energy (Eform) depends on the Ga chemical potential $\mu_{\mathrm{Ga}}[24,31]$ (Fig. 2(f)). For (100), the Ga-terminated interface is more stable than the As-terminated case, with relatively lower formation energy for most of the $\mu_{\mathrm{Ga}}$ range, shown by solid lines in Fig. 2(f) and experimentally [23]. For the Ga-rich case, Eform of Ga-terminated interface is 1.08 $\mathrm{eV} /$ cell lower than the As-terminated case. 
For the Ga-terminated (100) interface in Fig. 2(a), the Ga sites in the last GaAs layer (labelled as layer 2) remain tetrahedrally-coordinated. The As sites in the last YAs layer (layer 1) change from octahedral to 7 -fold coordinated. The $\mathrm{Y}$ sites in layer 1 change from octahedral to 5-fold coordinated. They either gained or lost one bond towards the GaAs side.

The (100) As-termination in Fig. 2(b) has two features. Firstly, the Y sites in layer 1 lie vertically above the interfacial As sites of the GaAs top layer (layer 2). These As sites have now reduced to a 3-fold coordination, while the $\mathrm{Y}$ sites in layer 1 remained 6-fold coordinated. However, the As site in layer 1 reduces to being 5 -fold coordinated. These miscoordinated atoms are significant for their unusual electronic structures.

Fig. 2(c) shows the nonpolar (110) face, where the Ga sites on the GaAs side retain their 4fold bonding, whereas the As sites become 5-fold, with two interfacial As-Y bonds per As atom. The Y sites on the YAs side retain their bulk octahedral coordination, while As sites become 5-fold coordinated with a dropped bond in the (010) plane. The YAs lattice is displaced relative to the GaAs lattice along the interface, with $\mathrm{Y}$ and As sites lying symmetrically above the interfacial Ga-As bond.

The partial density of states (PDOS) for these supercells is calculated. We find a very different behaviour compared to the MIGS model of elemental metals and semiconductors. The two (100) interfaces in Fig. 3(a) have a strong orientation dependence, with a p-type $\mathrm{SBH}$ of $0.16 \mathrm{eV}$ for As-termination and $1.36 \mathrm{eV}$ for Ga-termination, respectively. The SBHs were obtained using the core-level scheme [32,33], which takes the energy difference between VBM and core level to stay the same in either isolated bulk or interface material.

The key feature in the electronic structure that causes EF to vary with termination is the presence of strong localised interfacial gap states around EF. Previously, Delaney et al [24] considered the band alignments in terms of the electrostatic potential and the total valence charge densities. The gap states in the GaAs in contact with metals would normally be MIGS $[8,20]$. However, the states around $\mathrm{E}_{\mathrm{F}}$ at the $\mathrm{YAs} / \mathrm{GaAs}$ interface show very unusual behaviour. They are localized on the mis-coordinated atoms across the interface. These localized states decay on both the semiconductor side (as in MIGS) and the metal side (unlike MIGS). They are therefore 'defect-like states' in their localization, and similar in character to those seen previously in silicides $[15,16]$.

The 'defect states' occur at RE-As/GaAs interfaces due to the abrupt change in bonding from the zincblende GaAs lattice to the rocksalt YAs lattice. Although the As sublattice is continuous, the interface creates coordination defects on these sublattices. For the Asterminated interface, the defect states around $\mathrm{E}_{\mathrm{F}}$ are localized on the $\mathrm{p}-\pi$ states of 3 -fold As sites in layer 2, as well as the 5-fold As in layer 1, as in Fig. 4(a,b). The interface As-p orbital, with a PDOS peak at EF (Fig. 3(b)), pins EF close to the VBM, consistent with the general anion character of the p-orbital. We also built another As-terminated (100)YAs/GaAs, which is a shift of atomic positions of the one in our paper, and has 4-fold As in layer 2, 7-fold Y and 5-fold As in layer 1 (not shown). Its energy is $180 \mathrm{meV} / \mathrm{cell}$ higher than that in Fig. 2(b), but still has similar PDOS and EF pinning energy.

On the other hand, the defect state of the Ga-terminated (100) interface is localized on the 7-fold As sites in layer 1 and 4-fold Ga sites in layer 2, and this pins EF near the CBM, Fig. 4(c). The wavefunction around $\mathrm{EF}$ is not on the 5-fold $\mathrm{Y}$ site with its 'dangling bond', as 
mentioned in [23]. The cationic Ga-s states would normally have an energy close to CBM, while the anionic As-p states are close to the VBM. However, the PDOS calculation finds that the 7-fold As-sites in layer 1 have pushed these As states up in the GaAs gap to lie near the CBM. Hence, the pinning energy is near the CBM by defect states on As-sites as well as on Ga-sites, as seen in the PDOS, Fig. 3(c).

For the Ga-terminated (111) interface, the As sites in layer 1 are 6-fold coordinated, the same as in bulk YAs, but unlike the 4-fold As in bulk GaAs (Fig. 2(d)). We find interfacial defect gap states localised on Ga-sites in layer 2 and As sites in layer 3, Fig. 4(d). The defect states on As-sites are like for the (100)Ga-termination case, forced up towards the CBM. Together with defect states on Ga-sites, they pin $\mathrm{E}_{\mathrm{F}}$ at $1.0 \mathrm{eV}$ above VBM, Fig 3(d).

For the As-terminated (111) interface, the last As sites of the YAs lie directly above the last $\mathrm{Ga}$ sites of the GaAs. Here, there is a defect gap state localised on the As states, (Fig. 4(e)) and this pins EF at $0.05 \mathrm{eV}$ above the VBM, Fig. 3(d). Thus, the Fermi energies for each of these polar interfaces is explained by the energies of the associated interface defect gap states, not the CNLs. Thus the defect states have a sufficient density to pin $\mathrm{E}_{\mathrm{F}}$ at very different energies for the two terminations, much further apart than the $0.3 \mathrm{eV}$ separation that a dipole layer causes in other GaAs Schottky barriers [34].

For the nonpolar (110)YAs/GaAs interfaces, there are no defect gap states and $\mathrm{EF}_{\mathrm{F}}$ is pinned near midgap at $0.50 \mathrm{eV}$ by MIGS. There are no defect interface states because the interface involves no mis-coordinated sites, as shown in Fig. 2(c). Thus the (110) behaves like a Schottky barrier pinned by normal MIGS with a single pinning energy very close to the CNL energy of $0.54 \mathrm{eV}$ (Table 1). But for (100) and (111) interfaces, their mis-coordinated sites induce localised defect interface states, with a high local DOS. Their DOS dominate any underlying MIGS, and give very different pinning energies for the two different terminations.

Table 1. Calculated p-type SBHs for YAs/GaAs and ScN/GaN interfaces by GGA and SX functional, respectively.

\begin{tabular}{c|c|c|c}
\hline & $\begin{array}{c}\text { (100) Cation- } \\
\text { termination }\end{array}$ & $\begin{array}{c}\text { (100) Anion- } \\
\text { termination }\end{array}$ & (110) nonpolar \\
\hline $\mathrm{YAs} / \mathrm{GaAs}^{*}$ & 1.36 & 0.16 & 0.50 \\
\hline $\mathrm{ScN} / \mathrm{GaN}^{*}$ & 1.2 & 0.05 & 0.51 \\
\hline
\end{tabular}

${ }^{*} \mathrm{VBO}$ and ${ }^{\$} \mathrm{SX}$ functional for ScN/GaN.

We now consider the $\mathrm{ScN} / \mathrm{GaN}$ interface. Although rocksalt $\mathrm{ScN}$ is a semiconductor with a $1.3 \mathrm{eV}$ gap $[35,36]$ rather than a metal as YAs (Fig. 1), the ScN/GaN interfaces also possess interface 'defect gap states' which turn out to give band offsets which vary with termination. The $\mathrm{ScN} / \mathrm{GaN}$ interface is a type I band alignment. GGA functional gives a metal behaviour for $\mathrm{ScN}$, so the calculated PDOSs use the SX functional, which is also confirmed by HSE functional, as in Fig. 5. The distinct orientation-dependent EF pinning energies are shown in Fig. 5(a). A $1.2 \mathrm{eV}$ shift is a huge variation in VBO with termination.

The defect gap state for N-terminated interface lies on the interfacial N-sites and the VBM of $\mathrm{ScN}$ is pinned near VBM of GaN, as in the As-terminated GaAs interface in Fig. 4(a). This gives a VBO of $\sim 0 \mathrm{eV}$. In contrast, the SX PDOS of the Ga-terminated ScN/GaN interface is more complicated, with one peak around $\mathrm{E}_{\mathrm{F}}(\sim 2.7 \mathrm{eV}$ above GaN's VBM) and another peak at $\sim 1.9 \mathrm{eV}$ (Fig. 5(b)). This is caused by the relatively longer interfacial Ga-N bond $(2.75 \AA$ 
shown in Fig. 5c,d), much longer than the bulk Ga-N bond length of $2.0 \AA$. This causes its higher interface formation energy than the YAs/GaAs interface (dashed lines in Fig. 2(f)). Hence, both the $\mathrm{N}$-p orbitals in layer 1 and the Ga-s in layer 2 give a defect gap state lying near the $\mathrm{E}_{\mathrm{F}}$, as shown in Fig. 5(c). Hence, the band-edges of $\mathrm{ScN}$ are forced upward, giving a VBO of $\sim 1.2 \mathrm{eV}$, Fig. 5(a). The other peak lying $1.9 \mathrm{eV}$ above VBM is caused by the interfacial Ga-s and N-p orbitals, as shown by the wavefunction in Fig. 5(d). Thus, the band offsets in the YAs/GaAs and $\mathrm{ScN} / \mathrm{GaN}$ systems are both controlled by the anionic sublattice, as they control the anion $(\mathrm{As} / \mathrm{N})$ terminated interfaces directly, and for the cation $(\mathrm{Ga})$ terminated case, they set the SBH/VBO shift by the anion bonding.

The $\mathrm{ScN} / \mathrm{GaN}$ interface is of particular interest because of its large change in band offset with different terminations. This is controlled by nitrogen sites for both terminations, and it is interesting that $\mathrm{N}$ bonding can cause a pinning energy shift (i.e., $\mathrm{VBO}$ ) of $1.2 \mathrm{eV}$ from Ga- to $\mathrm{N}$-termination, following the same mechanism as in the YAs/GaAs case.

Thus, the ScN/GaN semiconductor interface behaves differently to typical semiconductor heterojunctions. Usually, their band offsets vary little with face orientation, and the main discussion is whether the band offset mainly follows an alignment with an absolute reference energy such as the vacuum level or a core level [37,38], or with the internal alignment of each semiconductor's CNLs $[38,39]$. When there is a dependence on face termination (under anion-rich or cation-rich ambient), it gives rise to a dipole layer and this usually causes a significant band offset variation which follows the valence charge distribution [40]. However, here the SBH or VBO shift arises mostly from different pinning energies due to the different defect gap states for each termination. It is unusual to be as large as $1.2 \mathrm{eV}$ in $\mathrm{ScN} / \mathrm{GaN}$. However, a disadvantage of this mechanism is that it adds gap states within the smaller gap of the type I alignment at the semiconductor interface, which is a disadvantage for electronic devices compared to normal alignment schemes [20,40]. The interface defect position and density could be experimentally measured by electrical or spectroscopy characterizations, but a quantitative comparison between the calculated results and experimental measurements is still difficult at the moment.

In conclusion, the MIGS model is generally applicable to many semiconductor/metal systems, but not all. We have identified the rare-earth arsenide/GaAs interface as one specific case where the standard MIGS model does not hold. It is found that the (100) interfaces with different terminations show obviously different Schottky barrier heights, thus different pinning energies. This is due to the mis-coordinated atoms at the interface. The states localize at the interface, and decay into both the semiconductor side and metal side. These observations are useful to help identify other systems with mis-coordinated interface sites so that we can tune the Fermi level, modify the Schottky barrier heights and thus design devices with lower contact resistances, which are needed for both highly scaled $\mathrm{Si}$ devices and modern 2D semiconductor systems.

Acknowledgements: The authors acknowledge funding from EPSRC grant EP/P005152/1. We also thank the support from Supercomputing Wales under the project SCW1070.

Data Availability Statement: The data that support the findings of this study are available from the corresponding author upon reasonable request. 


\section{Reference}

1. A. Allain, J. Kang, K. Banerjee, and A. Kis, Nat. Mater. 14, 1195 (2015).

2. S. Lee, A. Tang, S. Aloni, and H. S. Philip Wong, Nano Lett. 16, 276 (2015).

3. C. Kim, I. Moon, D. Lee, M. Choi, F. Ahmed, S. Nam, Y. Cho, H. Shin, S. Park, and W. Yoo, ACS Nano 11, 1588 (2017).

4. G. Kim, S. Kim, J. Park, K. H. Han, J. Kim, and H. Yu, ACS Nano 12, 6292 (2018)

5. Y. Liu, J. Guo, E. Zhu, L. Liao, S. Lee, M. Ding, I. Shakir, V. Gambin, Y. Huang, and X. Duan, Nature 557, 696 (2018).

6. J. Tersoff, Phys. Rev. Lett. 52, 465 (1984); Phys. Rev. B 32, 6968(R) (1985)

7. W. Monch, Phys. Rev. Lett. 58, 1260 (1987).

8. J. Robertson, J. Vac. Sci. Technol. B 18, 1785 (2000).

9. A. Dimoulas, P. Tsipas, A. Sotiropoulos, and E. K. Evangelou, Appl. Phys. Lett. 89, 252110 (2006).

10. L. Lin, Y. Guo and J. Robertson, Appl. Phys. Lett. 101, 052110 (2012).

11. H. Li, Y. Guo and J. Robertson, Sci. Rep. 7, 16669 (2017).

12. J. L. Freeouf, Solid State Commun. 33, 1059 (1980)

13. R. T. Tung, Phys. Rev. Lett. 52, 461 (1984).

14. T. Nishimura, T. Yajima and A. Toriumi, Appl. Phys. Express 9, 081201 (2016).

15. Z. Zhang, Y. Guo and J. Robertson, Under review (2020).

16. H. Fujitani and S. Asano, Phys. Rev. B 50, 8681 (1994).

17. W. E. Spicer, I. Lindau, P. Skeath, C. Y. Su, and P. Chye, Phys. Rev. Lett. 44, 420 (1980).

18.R. E. Allen, T. J. Humphreys, J. D. Dow, and O. F. Sankey, J. Vac. Sci. Tecnol. B 2, 449 (1984)

19.F. Flores, C. Tejedor, J. Phys. C: Solid State Phys. 20, 145 (1987).

20. J. Robertson, Appl. Phys. Lett. 94, 152104 (2009).

21. C. J. Palmstrøm, T. L. Cheeks, H. L. Gilchrist, J. G. Zhu, C. B. Carter, B. J. Wilkens, and R. Martin, J. Vac. Sci. Technol. A 10, 1946 (1992).

22. D. O. Klenov, J. M. Zide, J. D. Zimmerman, A. C. Gossard, and S. Stemmer, Appl. Phys. Lett. 86, 241901 (2005).

23. W. R. L. Lambrecht, A. G. Petukhov and B. T. Hemmelman, Solid State Commun. 108, 361 (1998).

24. K. T. Delaney, N. A. Spaldin and C. G. Van de Walle, Phys. Rev. B 81, 165312 (2010).

25. A. Guivarc H, Y. Ballini, M. Minier, B. Guenais, G. Dupas, G. Ropars, and A. Regreny, J. Appl. Phys. 73 , 8221 (1993).

26. J. D. Zimmerman, E. R. Brown and A. C. Gossard, J. Vac. Sci. Technol. B 23, 1929 (2005); M. P. Hanson, S. R. Bank, J. M. O. Zide, J. D. Zimmerman, and A. C. Gossard, J. Cryst. Growth 301, 4 (2007).

27. S. Khalid, A. Sharan and A. Janotti, Phys. Rev. B 101, 125105 (2020).

28. S. J. Clark, M. D. Segall, C. J. Pickard, P. J. Hasnip, M. J. Probert, K. Refson, and M. C. Payne, Z. Kristallogr. 220, 567 (2005).

29. S. J. Clark and J. Robertson, Phys. Rev. B 82, 085208 (2010).

30. R. Gillen, S. J. Clark, and J. Robertson, Phys. Rev. B 87, 125116 (2013)

31. Z. Zhang, R. Cao, C. Wang, H. Li, H. Dong, W. Wang, F. Lu, Y. Cheng, X. Xie, H. Liu, K. Cho, R. Wallace, and W. Wang, ACS Appl. Mater. Interfaces 7, 5141 (2015).

32. E. A. Kraut, R. W. Grant, J. R. Waldrop, and S. P. Kowalczyk, Phys. Rev. Lett. 44, 1620 (1980).

33. Z. Zhang, Y. Guo and J. Robertson, Appl. Phys. Lett. 114, 161601 (2019).

34. C. Berthod, N. Binggeli and A. Baldereschi, Phys. Rev. B 68, 085323 (2003).

35. D. Gall, M. Städele, K. Järrendahl, I. Petrov, P. Desjardins, R. T. Haasch, T. Y. Lee, and J. E. Greene, Phys. Rev. B 63, 125119 (2001);

36. C. Stampfl, W. Mannstadt, R. Asahi, and A. J. Freeman, Phys. Rev. B 63, 155106 (2001)

37. S. H. Wei and A. Zunger, Appl. Phys. Lett. 63, 2549 (1993).

38. Y. Hinuma, A. Gruneis, G. Kresse, and F. Oba, Phys. Rev. B 90, 155405 (2014).

39. Y. Guo, H. Li, S. J. Clark, and J. Robertson, J. Phys. Chem. C 123, 5562 (2019).

40. J. Tersoff, Phys. Rev. B 30, 4874(R) (1984). 

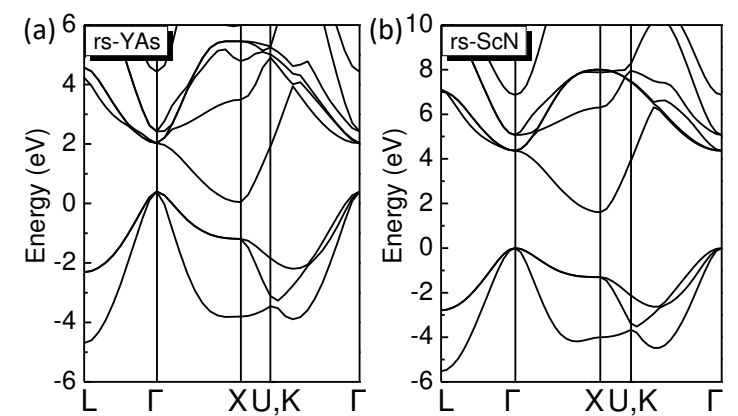

Figure 1. SX band structures for (a) YAs and (b) ScN bulk, respectively. YAs is a metal while ScN is a semiconductor with an indirect gap of $1.53 \mathrm{eV}$, close to the experimental gap of $1.3 \pm 0.3 \mathrm{eV}$.

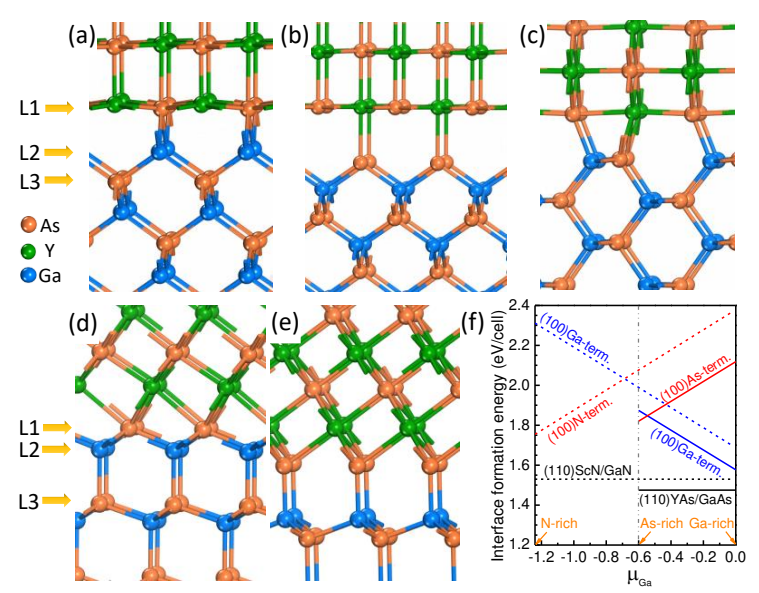

Figure 2. Atomic structures for YAs/GaAs (a) (100)Ga-termination, (b) (100)As-termination, (c) (110) nonpolar, (d) (111)Ga-termination and (e) (111)As-termination interfaces, respectively. The interfacial atomic layers are labelled as L1, L2, L3. (f) shows the interface formation energy, where the solid lines represent $\mathrm{YAs} / \mathrm{GaAs}$, and dashed lines represent $\mathrm{ScN} / \mathrm{GaN}$ interfaces, respectively. 

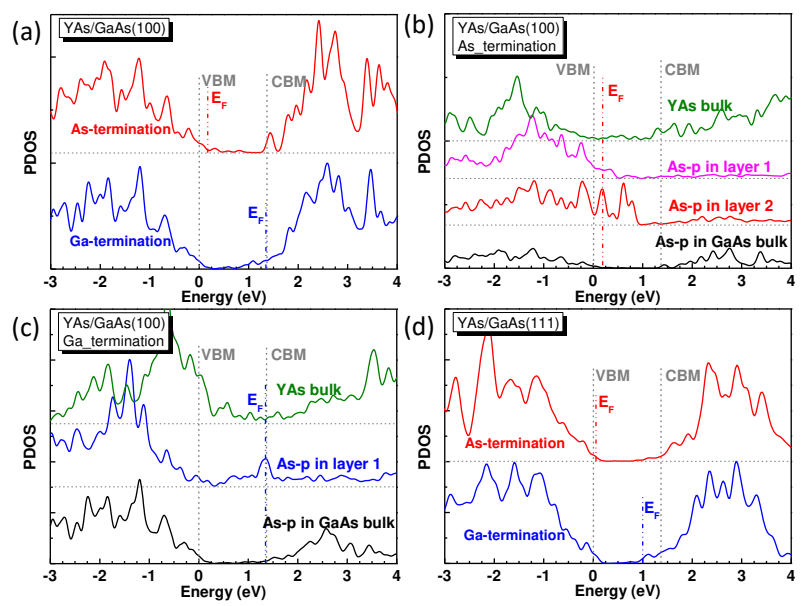

Figure 3. (a) PDOS of GaAs bulk atoms away from the interface in (100)YAs/GaAs. (b) and (c) are As-p PDOS comparison for two different terminations. (d) PDOS of (111)YAs/GaAs interfaces. $\mathrm{VBM}$ is set to $0 \mathrm{eV}$ and $\mathrm{E}_{\mathrm{F}}$ is labelled individually.
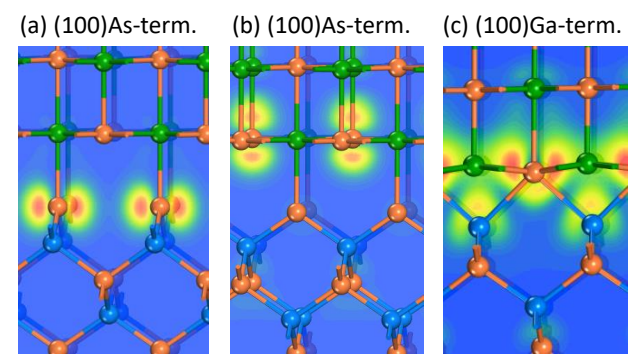

(d) (111)Ga-term.

(e) (111)As-term.
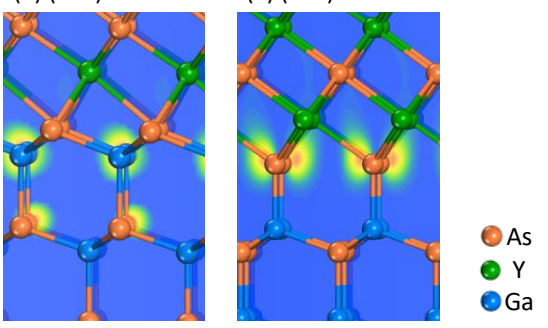

Figure 4. Wavefunction around $\mathrm{E}_{\mathrm{F}}$ for $\mathrm{YAs} / \mathrm{GaAs}$. (a) and (b) are two different orbitals around $\mathrm{E}_{\mathrm{F}}$ for different viewing angles for (100) As-termination, with localized wavefunction on interfacial 3-fold (layer 2) and 5-fold (layer 1) As atoms, respectively. (c) is (100)Ga-terminated interface. (d) and (e) are (111)Ga- and As-terminations, respectively. 


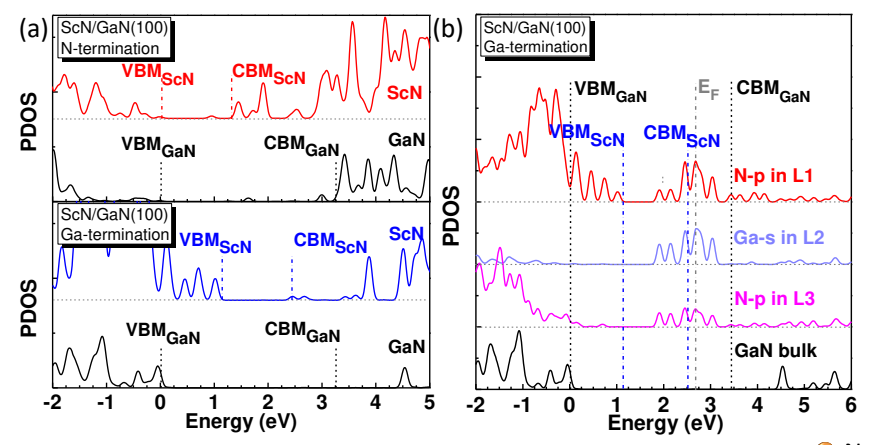

(c)
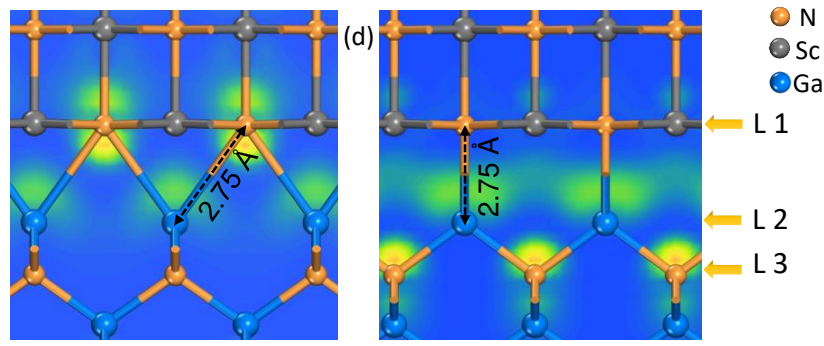

Figure 5. (a) SX PDOS of GaN and $\mathrm{ScN}$ bulk atoms away from the interface in (100)ScN/GaN. (b) PDOS comparison between the GaN bulk and the interfacial atoms in Ga-terminated interface. The VBM of GaN bulk is aligned to $0 \mathrm{eV}$. (c) and (d) are two different orbitals around $\mathrm{E}_{\mathrm{F}}(\sim 2.7 \mathrm{eV}$ above GaN's VBM) and the PDOS peak at $1.9 \mathrm{eV}$ above VBM for Ga-terminated interface, respectively. 

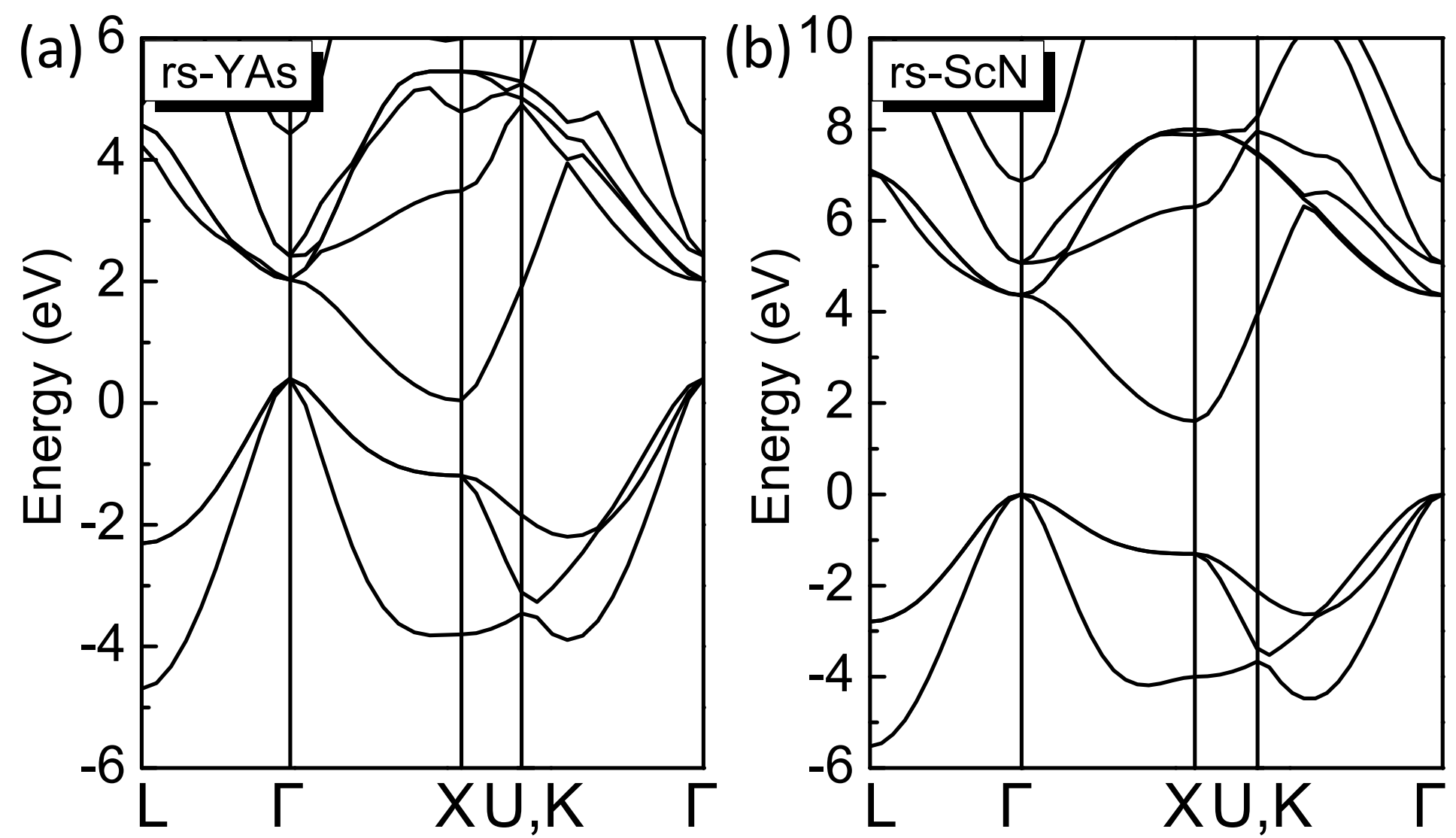
This is the author's peer reviewed, accepted manuscript. However, the online version of record will be different from this version once it has been copyedited and typeset. PLEASE CITE THIS ARTICLE AS DOI: 10.1063/5.0007479
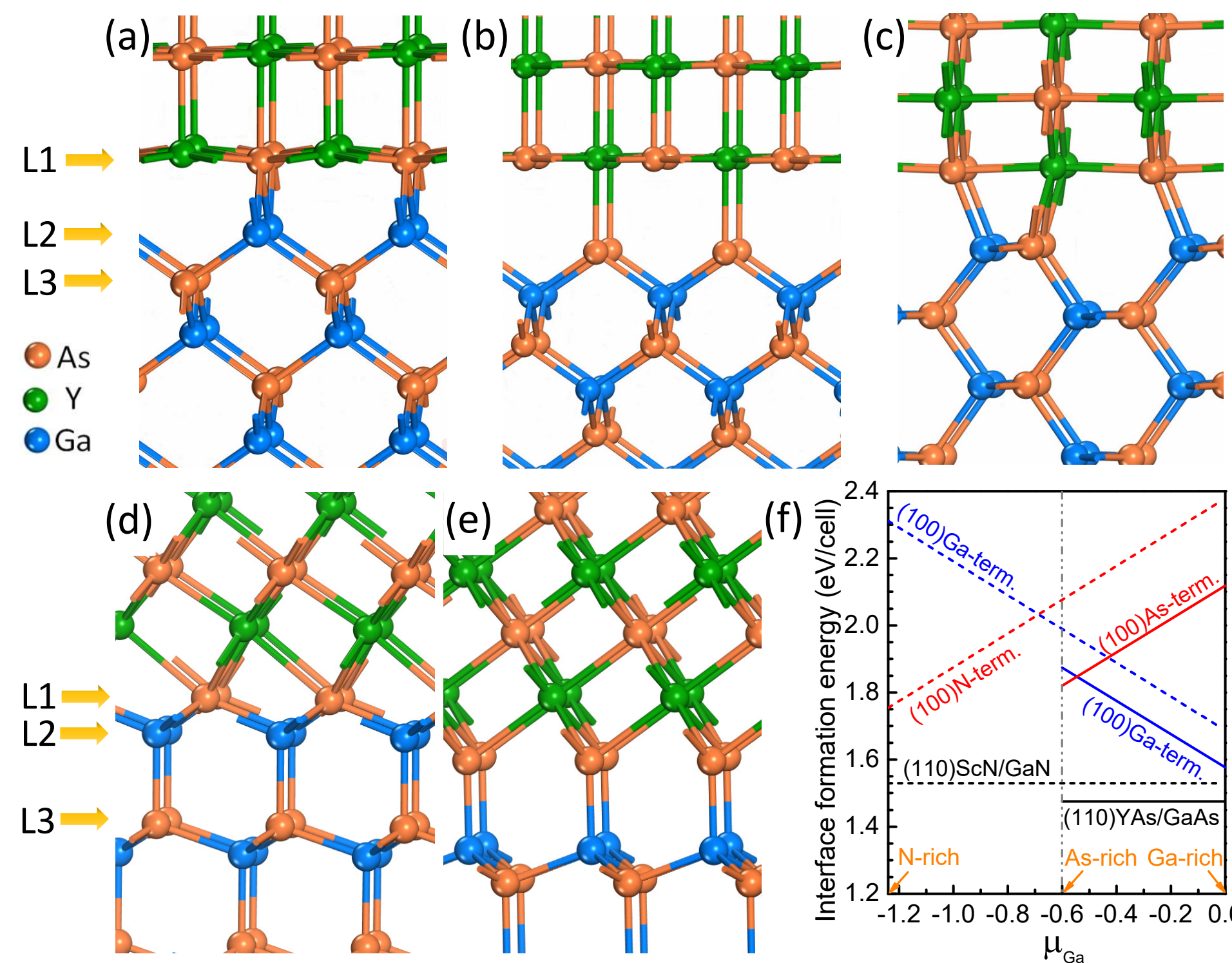

(f) $\widehat{\overline{\bar{d}}}$

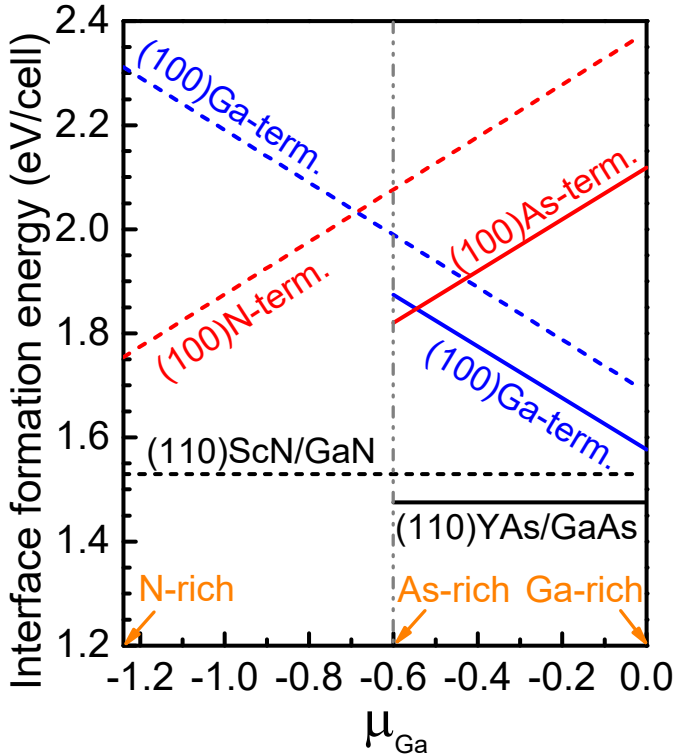


This is the author's peer reviewed, accepted manuscript. However, the online version of record will be different from this version once it has been copyedited and typeset.

PLEASE CITE THIS ARTICLE AS DOI: 10.1063/5.0007479
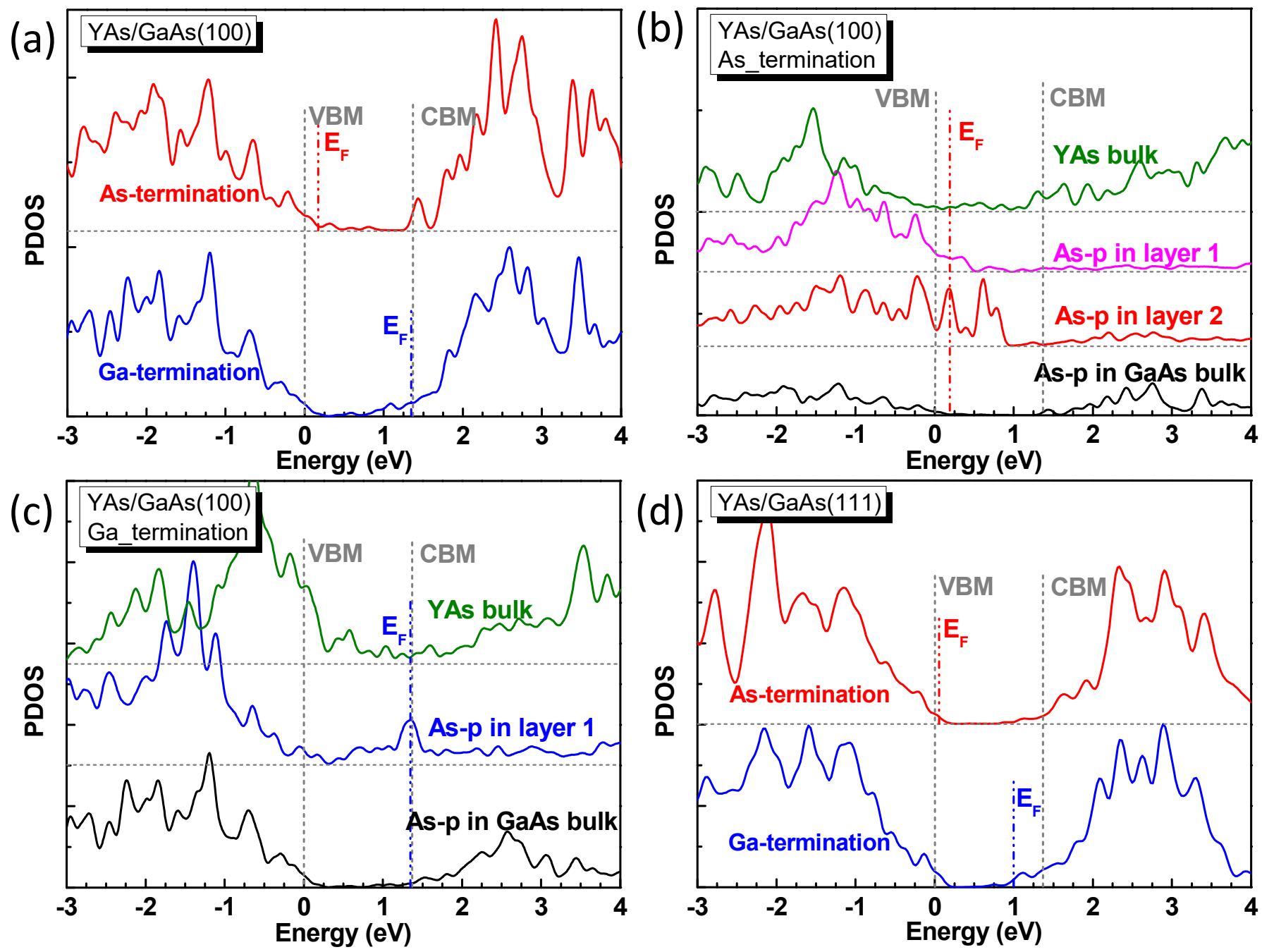
This is the author's peer reviewed, accepted manuscript. However, the online version of record will be different from this version once it has been copyedited and typeset. PLEASE CITE THIS ARTICLE AS DOI: 10.1063/5.0007479

(a) (100)As-term.

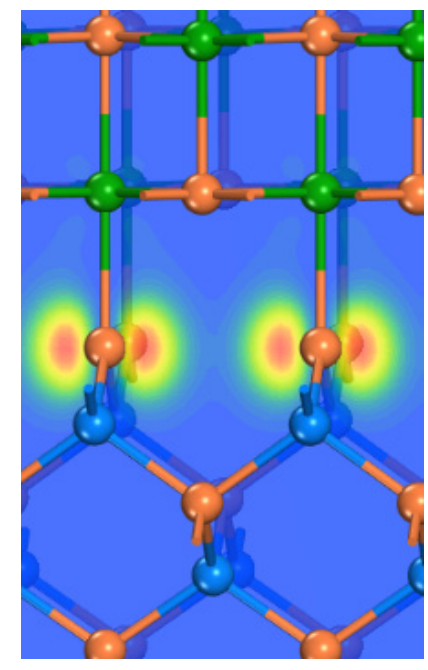

(d) (111)Ga-term.

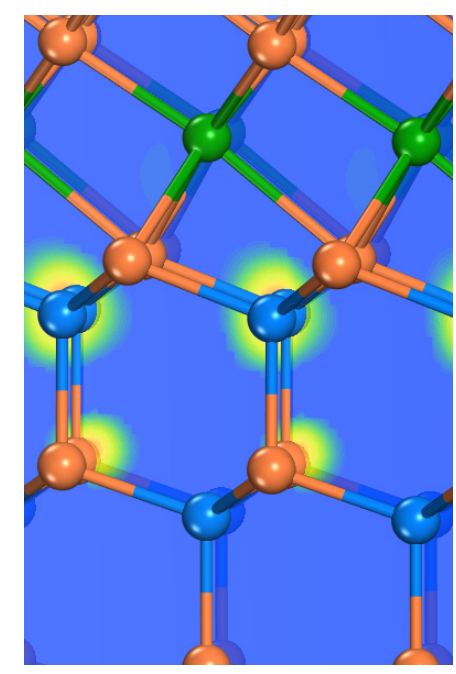

(b) (100)As-term.

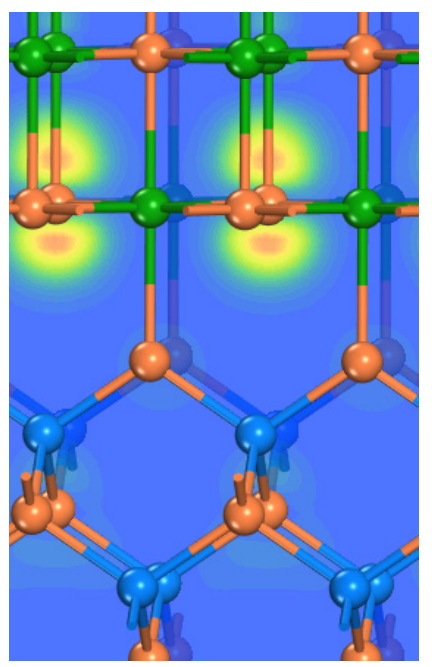

(e) (111)As-term.

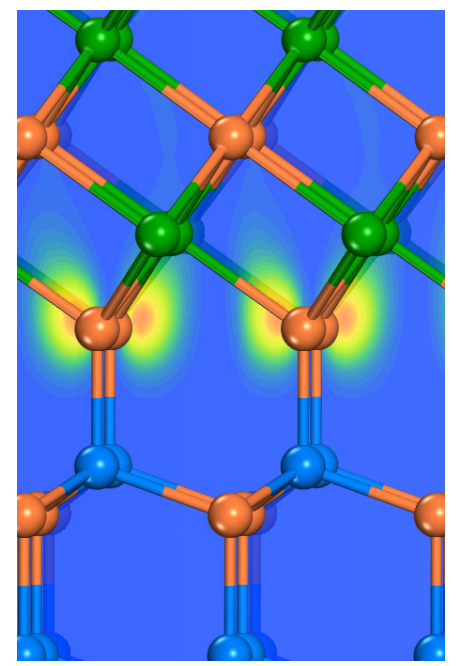

- As

- $Y$

- Ga 

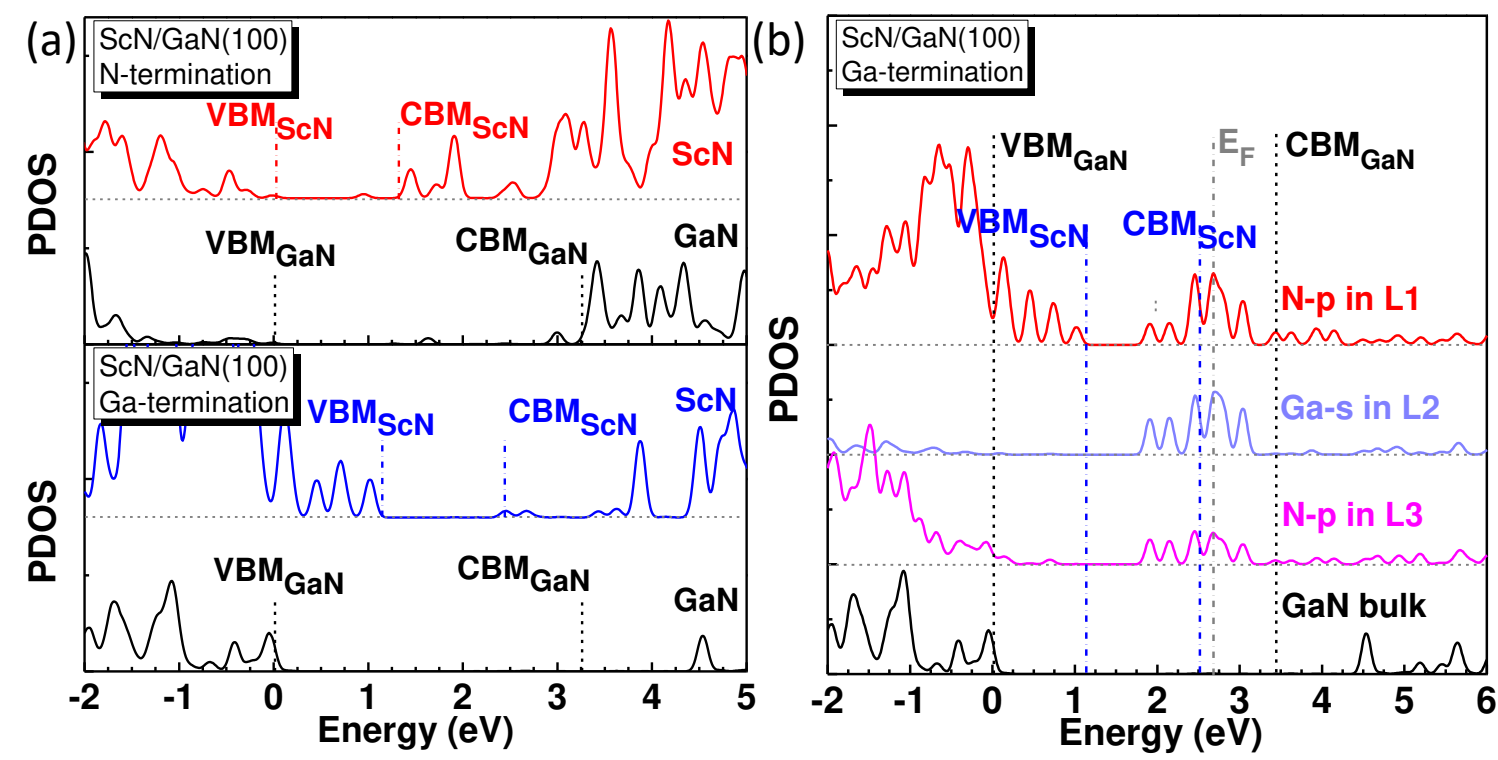

(c)

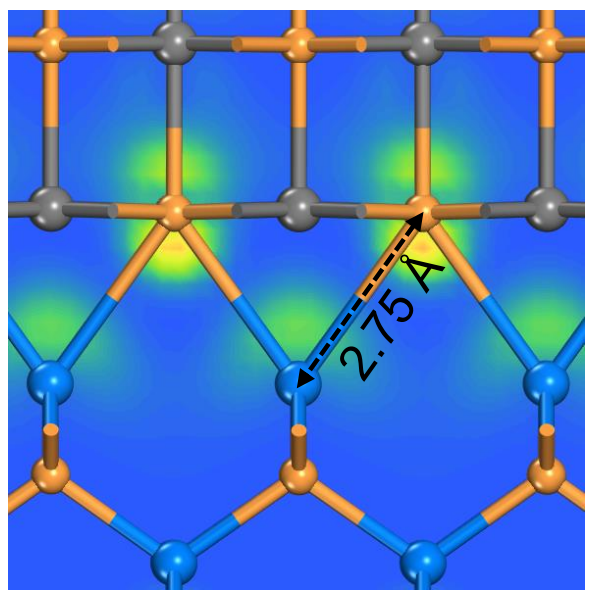

(d)

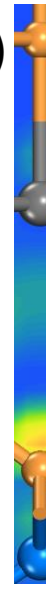

- N

- $S c$ - Ga 